\title{
Composite learning adaptive backstepping control using neural networks with compact supports
}

\author{
Yongping Pan ${ }^{\mathrm{a}, \mathrm{b}}$, Chenguang Yang ${ }^{\mathrm{c}}$, Mahardhika Pratama ${ }^{\mathrm{d}}$, \\ Haoyong $\mathrm{Yu}^{\mathrm{b}, \star}$ \\ ${ }^{a}$ National University of Singapore (Suzhou) Research Institute, Suzhou, China \\ ${ }^{\mathrm{b}}$ Department of Biomedical Engineering, National University of Singapore, \\ Singapore \\ ${ }^{\mathrm{c}}$ Bristol Robotics Laboratory, University of the West of England, Bristol, UK \\ ${ }^{\mathrm{d}}$ School of Computer Science and Engineering, Nanyang Technological University, \\ Singapore
}

\begin{abstract}
The ability to learn is crucial for neural network (NN) control as it is able to enhance the overall stability and robustness of control systems. In this study, a composite learning control strategy is proposed for a class of strict-feedback nonlinear systems with mismatched uncertainties, where raised-cosine radial basis function (RCRBF)NNs with compact supports are applied to approximate system uncertainties. Both online historical data and instantaneous data are utilized to update NN weights. Practical exponential stability of the closed-loop system is established under a weak excitation condition termed interval excitation (IE). The proposed approach ensures fast parameter convergence, implying an exact estimation of plant uncertainties, without the trajectory of NN inputs being recurrent and the time derivation of plant states. The RCRBF-NNs applied not only reduces computational cost, but also facilitates the exact determination of a subregressor activated along any trajectory of NN inputs so that the IE condition is verifiable. Numerical results have verified validity and superiority of the proposed approach.
\end{abstract}

Key words: Adaptive control, composite learning, backstepping, neural network, parameter convergence.

ऋ Corresponding author: H. Yu.

Email address: bieyhy@nus.edu.sg (Haoyong Yu). 


\section{Introduction}

One of the successful stories of applying machine learning to intelligent control is neural network (NN)-based adaptive control (NNAC) [1]. Compared with the traditional adaptive control, the most appealing merit of NNAC is that the modelling difficulty in many practical control problems can be greatly mitigated resulting in the simplification of control synthesis for a wider class of nonlinear systems with functional uncertainties [2]. However, in most existing NNAC methods, e.g. see [3-17] for some recent results, the ability of NNs to learn plant uncertainties is not completely exploited and only tracking error convergence is available. The ability to learn for NNs, reflected by the convergence of $\mathrm{NN}$ weights, is guaranteed by the well-known condition termed persistent excitation (PE) [18]. Parameter convergence in NNAC brings several salient benefits, e.g. accurate online modeling, superior tracking, and robustness against various perturbations [19].

The classical PE condition is too stringent and often infeasible in practice [20]. A more practical PE condition based on radial basis function (RBF)NNs shows that any recurrent trajectory of NN inputs that stays within a regular lattice leads to a partial PE condition [21]. Based on the practical PE condition, several NN learning control (NNLC) methods were proposed to guarantee closed-loop practical exponential stability so that accurate NN learning is obtainable [21-25]. The relationship between PE levels and RBFNN structures was analyzed in [26]. However, in the existing NNLC methods, the necessity that the trajectory of $\mathrm{NN}$ inputs is recurrent is still stringent in practice, and the parameter convergence rate highly depends on PE levels, which generally gives rise to a slow parameter convergence speed [27].

A hybrid direct and indirect adaptive control strategy termed composite adaptive control utilizes both tracking and prediction errors to update parameter estimates such that both tracking accuracy and parameter convergence can be improved [28-30]. Motivated by the composite adaptation, an emerging composite learning technique was proposed to achieve parameter convergence in adaptive control at the absence of PE [31-37]. The difference of the composite learning compared with the composite adaptation is that online historical data (OHD) are employed to construct prediction errors so that closedloop exponential stability is ensured by an interval excitation (IE) condition which greatly relaxes the $\mathrm{PE}$ condition. A model reference composite learning control method was presented for a class of nonlinear systems with matched parametric uncertainties in [31], where the time derivation of plant states is eliminated by using an integral transformation. In [32], the approach of [31] was extended to a class of strict-feedback nonlinear systems with mismatched parametric uncertainties via command filtered backstepping. The approach of [32] was further extended to the case with functional uncertainties in [33]. 
In [34], the composite learning was applied to achieve parameter convergence in least squares-based identification and indirect adaptive control. The IE condition for parameter convergence in the composite learning was relaxed to be a condition of sufficient excitation (SE) in [35]. In [36], a composite learning control approach was developed for a general class of robotic arms. In [37], an NN composite learning control approach with friction compensation was designed and implemented to an industrial robot arm. However, the approaches of $[36,37]$ are specifically designed for robotic systems, the time derivatives of plant states are needed to be estimated in $[32,33]$, and the extension of the integral transformation in $[31,34,35]$ to the case with mismatched uncertainties is infeasible.

In this article, an NN composite learning control (NNCLC) strategy is presented for the class of strict-feedback nonlinear systems in [29], where raised-cosine $\mathrm{RBF}$ (RCRBF)-NNs are used to approximate plant uncertainties. Command filtered backstepping [38] is resorted to alleviate the problem of "explosion of complexity" in the traditional integrator backstepping. Compared with existing NNLC approaches, the attractive feature of our approach is that fast parameter convergence in NNs, implying exact learning of plant uncertainties, is guaranteed without the trajectory of NN inputs being recurrent. Compared with the NNCLC approach of [33], the distinctive feature of the proposed approach include: 1) The state derivation is not needed for the computation of prediction errors; 2) the RCRBF-NNs applied is not only helpful for reducing computational cost but also convenient for exactly determining a subregressor activated along any trajectory of NN inputs so that the IE condition is verifiable.

The rest of this article is organized as follows: The problem is formulated in Section II; the RCRBF-NN is described in Section III; the NNCLC is designed in Section IV; illustrative results are provided in Section V; conclusions are drawn in Section VI. Through out this brief, $\mathbb{R}, \mathbb{R}^{+}$and $\mathbb{R}^{n}$ denote the spaces of real numbers, positive real numbers and real $n$-vectors, respectively, $L_{\infty}$ is the space of bounded signals, $\|\mathbf{x}\|$ is the Euclidean norm of $\mathbf{x}, \min \{\cdot\}$, $\max \{\cdot\}$ and $\sup \{\cdot\}$ are the operators of minimum, maximum and supremum, respectively, $\tanh (x)$ is a hyperbolic tangent function, $\Omega_{c}:=\{\mathbf{x} \mid\|\mathbf{x}\| \leq c\}$ is the ball of radius $c$, and $\mathcal{C}^{k}$ represents the space of functions for which all $k$-order derivatives exist and are continuous, where $c \in \mathbb{R}^{+}, x \in \mathbb{R}, \mathbf{x} \in \mathbb{R}^{n}$, and $n$ and $k$ are positive integers. 


\section{Problem Formulation}

Consider the following class of $n$ th-order strict-feedback nonlinear systems with functional uncertainties [29]:

$$
\left\{\begin{array}{l}
\dot{x}_{i}=f_{i}\left(\mathbf{x}_{i}\right)+x_{i+1}, i=1 \text { to } n-1 \\
\dot{x}_{n}=f_{n}\left(\mathbf{x}_{n}\right)+u \\
y=x_{1}
\end{array}\right.
$$

with $\mathbf{x}_{i}(t):=\left[x_{1}(t), x_{2}(t), \cdots, x_{i}(t)\right]^{T} \in \mathbb{R}^{i}$, where $\mathbf{x}(t)=\mathbf{x}_{n}(t)$ is a vector of system states, $u(t) \in \mathbb{R}$ is a control input, $y(t) \in \mathbb{R}$ is a controlled output, $f_{i}\left(\mathbf{x}_{i}\right): \mathbb{R}^{i} \mapsto \mathbb{R}$ are unknown functions, and $i=1$ to $n$. Let $x_{d}(t) \in \mathbb{R}$ denote a desired output. The following assumptions are given to facilitate the control design.

Assumption 1 [41]: $f_{i}\left(\mathbf{x}_{i}\right)$ are of $\mathcal{C}^{1}$ for $i=1$ to $n$.

Assumption 2 [41]: $x_{d}(t)$ and $\dot{x}_{d}(t)$ are continuous and of $L_{\infty}$.

Let $\alpha_{i}(t) \in \mathbb{R}$ and $\alpha_{i}^{c}(t) \in \mathbb{R}$ with $i=1$ to $n-1$ be virtual control inputs and the filtered counterparts, respectively. Define tracking errors $e_{i}(t):=x_{i}(t)-$ $\alpha_{i-1}^{c}(t)$ with $\alpha_{0}^{c}(t)=x_{d}(t)$ and $i=1$ to $n$. Let $\mathbf{e}(t):=\left[e_{1}(t), e_{2}(t), \cdots, e_{n}(t)\right]^{T}$ and $\mathbf{x}_{d}(t):=\left[x_{d}(t), \dot{x}_{d}(t)\right]^{T} \in \Omega_{d} \subset \mathbb{R}^{2}$. In this study, the objective is to design an NN-based control law for the system (1) under Assumptions 1 and 2 such that the tracking error e tends to $\mathbf{0}$ and $f_{i}\left(\mathbf{x}_{i}\right)$ with $i=1$ to $n$ are accurately approximated by NNs along $\mathrm{NN}$ input trajectories.

\section{Radial-Basis-Function Neural Network}

Let $\Omega_{x} \in \mathbb{R}^{n}$ be a domain of $\mathrm{NN}$ approximation. The region of each $x_{i}$ is divided into $m_{i}-1$ uniform and symmetric grids with widths $\sigma_{i} \in \mathbb{R}^{+}$by $m_{i}$ grid points $c_{i}^{l_{i}} \in \mathbb{R}$, where $m_{i} \geq 3$ is an odd number, $l_{i}=1$ to $m_{i}$, and $i=1$ to $n$. Then, a RCRBF of the form [43]:

$$
\xi_{i}^{l_{i}}\left(x_{i}\right)=\left\{\begin{array}{l}
\frac{1}{2}\left(1+\cos \left(\frac{\pi\left(x_{i}-c_{i}^{l_{i}}\right)}{\sigma_{i}}\right)\right), \text { if }\left|x_{i}-c_{i}^{l_{i}}\right| \leq \sigma_{i} \\
0, \text { otherwise }
\end{array}\right.
$$

is applied to cover at least one grid for each possible $l_{i}$ and $i$. Hence, $N=$ $m_{1} m_{2} \cdots m_{n}$ neural nodes can be generated. The $N$ neural nodes ordered in an $n$-dimension matrix can be reordered into a one-dimension array through 
a scalar index

$$
\begin{aligned}
j= & l_{1}+m_{1}\left(l_{2}-1\right)+m_{1} m_{2}\left(l_{3}-1\right) \\
& +\cdots+m_{1} m_{2} \cdots m_{n-1}\left(l_{n}-1\right) .
\end{aligned}
$$

Then, a RCRBF-NN is represented as follows:

$$
\hat{f}(\mathbf{x}, \hat{W})=\Phi^{T}(\mathbf{x}) \hat{W}
$$

in which $\hat{W} \in \Omega_{w} \subset \mathbb{R}^{N}$ is a vector of $\mathrm{NN}$ weights, $\Phi(\mathbf{x})=\left[\phi_{1}(\mathbf{x}), \phi_{2}(\mathbf{x})\right.$, $\left.\cdots, \phi_{N}(\mathbf{x})\right]^{T} \in \mathbb{R}^{N}$ is a regressor, $\phi_{j}(\mathbf{x}):=\sum_{i=1}^{n} \xi_{i}^{l_{i}}\left(x_{i}\right)$ is a regression function corresponding to the th $j$ NN node, and $j=1$ to $N$. The RCRBF belongs to a class of localized RBFs as its support is a compact set $\left[c_{i}^{l_{i}}-\sigma_{i}, c_{i}^{l_{i}}+\sigma_{i}\right]$.

The RBF NN (2) is used to approximate a certain function $f(\mathbf{x}): \Omega_{x} \mapsto \mathbb{R}$ resulting in an optimal NN approximation error

$$
\varepsilon(\mathbf{x}):=f(\mathbf{x})-\hat{f}\left(\mathbf{x}, W^{*}\right)
$$

with $W^{*} \in \Omega_{w}$ a constant vector of optimal weights given by

$$
W^{*}:=\underset{\hat{W} \in \Omega_{w}}{\arg \min }\left\{\sup _{\mathbf{x} \in \Omega_{x}}|f(\mathbf{x})-\hat{f}(\mathbf{x}, \hat{W})|\right\} .
$$

The approximation theorem of RBF-NNs shows that $|\varepsilon(\mathbf{x})| \leq \varepsilon^{*}, \forall \mathbf{x} \in \Omega_{x}$ can be guaranteed for any given small constant $\varepsilon^{*} \in \mathbb{R}^{+}$if $N$ is sufficiently large [44]. The following definitions and lemmas are presented for the subsequent development.

Definition 1 [18]: A bounded signal $\Phi(t) \in \mathbb{R}^{N}$ is of IE over $\left[T_{e}-\tau_{d}, T_{e}\right]$ if $\exists T_{e}, \tau_{d}, \sigma \in \mathbb{R}^{+}$such that $\int_{T_{e}-\tau_{d}}^{T_{e}} \Phi(\tau) \Phi^{T}(\tau) d \tau \geq \sigma I$.

Definition 2 [18]: A bounded signal $\Phi(t) \in \mathbb{R}^{N}$ is of PE if $\exists \tau_{d}, \sigma \in \mathbb{R}^{+}$such that $\int_{t-\tau_{d}}^{t} \Phi(\tau) \Phi^{T}(\tau) d \tau \geq \sigma I, \forall t \geq 0$.

Lemma 1 [21]: For any given trajectory $\mathbf{x}(t): \mathbb{R}^{+} \mapsto \Omega_{x}, f(\mathbf{x})$ can be approximated by the RCRBF-NN (2) with a limited number of NN nodes located in a local region along $\mathbf{x}(t)$ such that ${ }^{1}$

$$
f(\mathbf{x})=\Phi_{\zeta}^{T}(\mathbf{x}) W_{\zeta}^{*}+\varepsilon(\mathbf{x})
$$

with $W_{\zeta}^{*} \in \mathbb{R}^{N_{\zeta}}$ and $\Phi_{\zeta} \in \mathbb{R}^{N_{\zeta}}$ subvectors of $W^{*}$ and $\Phi$, respectively, where $N_{\zeta}<N$ is the number of total activated NN nodes.

1 In [21], due to the usage of Gaussian RBFs, one has $f(\mathbf{x})=\Phi_{\zeta}^{T}(\mathbf{x}) W_{\zeta}^{*}+\varepsilon_{\zeta}(\mathbf{x})$ with $\varepsilon_{\zeta} \in \mathbb{R}^{+}$of the order $\varepsilon$. For the RCRBF-NN (2), as the RCRBF has a compact support, the outputs of most regression functions $\phi_{j}$ can be strictly zero, and thus, $\varepsilon$ can be used directly instead of $\varepsilon_{\zeta}$ in (5). 
Lemma 2 [21]: For the RBF NN (5) with centers placed on a regular lattice to cover $\Omega_{x}$, given any $\mathcal{C}^{1}$ recurrent trajectory $\mathbf{x}(t): \mathbb{R}^{+} \mapsto \Omega_{x}$, the subregressor $\Phi_{\zeta}(\mathbf{x})$ is almost always PE.

Remark 1: Because the RCRBF has a compact support, the maximal number of RCRBFs with nonzero values for a given input $x_{i}$, denoted by $\bar{m}$, is controlled by the width $\sigma_{i}$, and the number of current activated NN nodes in the RCRBF-NN (2) is at most $N_{c}=\bar{m}^{n}$. Typically, $\bar{m}$ is set to be 2 or 3 which is smaller than the numbers of grid points $m_{1}$ to $m_{n}$, and thus, $N_{c}$ is generally much smaller than the total number of NN nodes $N$. A distribution of RCRBFs is illustrated in Fig. 1, where $x_{i} \in[-3,3], m_{i}=7, \sigma_{i}=1.5, c_{i}^{l_{i}}$ $=l_{i}-4, \bar{m}=3, i=1$ to 3 , and $l_{i}=1$ to 7 . In this case, one has $N_{c}=$ $3^{3}=27$ which is much smaller than $N=7^{3}=343$. Therefore, we can use only activated NN nodes, determined by nonzero RCRBFs, to update $\hat{W}$ and to compute the NN output so that the RCRBF-NN (2) can have much lower computational cost than other types of RBF NNs [43].

\section{Composite Learning Backstepping Control}

\subsection{Neural network-based backstepping control}

In the subsequent sections, the valuation of all $i$ is $i=1$ to $n$ except special indications. As $f_{i}\left(\mathbf{x}_{i}\right)$ in (1) is unknown, a RCRBF-NN of the form (2) with extra subscripts $i$ as follows:

$$
\hat{f}_{i}\left(\mathbf{x}_{i}, \hat{W}_{i}\right)=\Phi_{i}^{T}\left(\mathbf{x}_{i}\right) \hat{W}_{i}
$$

is applied to approximate each $f_{i}\left(\mathbf{x}_{i}\right)$, where $\hat{W}_{i} \in \Omega_{w_{i}} \subset \mathbb{R}^{N_{i}}, \Phi_{i} \in \mathbb{R}^{N_{i}}$, $\left\|\Phi_{i}\left(\mathbf{x}_{i}\right)\right\| \leq \psi_{i}[18], N_{i}$ is the number of neural nodes, and $\psi_{i} \in \mathbb{R}^{+}$is a constant. An NN-based command-filtered backstepping control law is presented

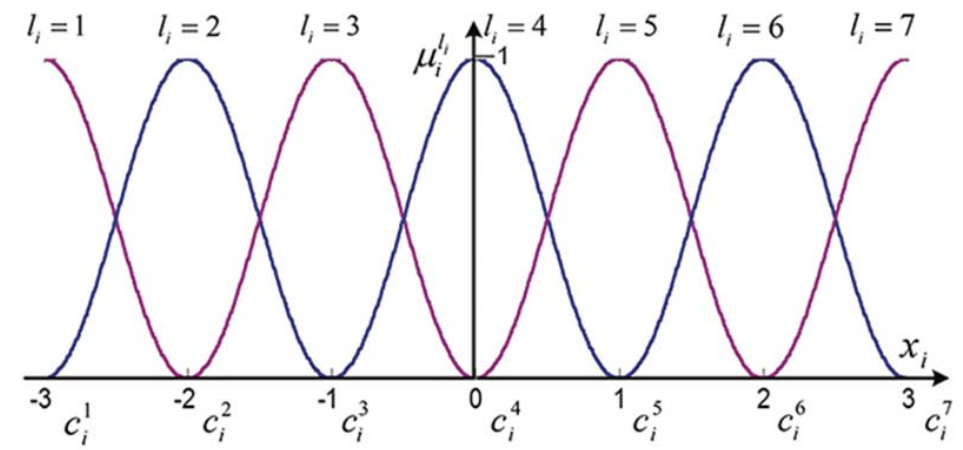

Fig. 1. A distribution of raised-cosine radial basis functions. 
as follows:

$$
\left\{\begin{array}{l}
\alpha_{i}=v_{i}+\dot{\alpha}_{i-1}^{c}-\Phi_{\zeta i}^{T}\left(\mathbf{x}_{i}\right) \hat{W}_{\zeta i} \\
(i=1,2, \cdots, n-1) \\
u=v_{n}+\dot{\alpha}_{n-1}^{c}-\Phi_{\zeta n}^{T}\left(\mathbf{x}_{n}\right) \hat{W}_{\zeta n}
\end{array}\right.
$$

where $v_{i} \in \mathbb{R}$ is an auxiliary control term given by

$$
v_{i}=-k_{i} e_{i}-\beta_{i} \tanh \left(e_{i} / v_{i}\right)
$$

with $k_{i}, \beta_{i}, v_{i} \in \mathbb{R}^{+}$being control parameters, and $\alpha_{i}^{c}$ and $\dot{\alpha}_{i}^{c}$ with $i=1$ to $n-$ 1 are generated by the command filter as follows [38]:

$$
\left\{\begin{array}{l}
\dot{z}_{1}=z_{2} \\
\dot{z}_{2}=-2 \varsigma \omega z_{2}+\omega^{2}\left(\alpha_{i}-z_{1}\right)
\end{array}\right.
$$

with $z_{1}(0)=\alpha_{i}(0), z_{2}(0)=0, \alpha_{i}^{c}=z_{1}$, and $\dot{\alpha}_{i}^{c}=z_{2}$, where $\omega \in \mathbb{R}^{+}$is a natural frequency, and $\varsigma \in \mathbb{R}^{+}$is a damping ratio. Note that only excited neural nodes are applied to compute the $\mathrm{NN}$ output in (7), and $\tanh \left(e_{i} / v_{i}\right)$ in (8) serves as an approximation of the sliding mode control term $\operatorname{sgn}\left(e_{i}\right)$ to reject system perturbations.

It follows from Lemma 1 that $f_{i}\left(\mathbf{x}_{i}\right)$ can be expressed by

$$
f_{i}\left(\mathbf{x}_{i}\right)=\Phi_{\zeta i}^{T}\left(\mathbf{x}_{i}\right) W_{\zeta i}^{*}+\varepsilon_{i}\left(\mathbf{x}_{i}\right)
$$

where $W_{\zeta i}^{*}$ is a subvector of $W_{i}^{*}$, and $\varepsilon_{i}$ and $W_{i}^{*}$ are given by (3) and (4) with extra subscripts $i$, respectively. Thus, one has $\left|\varepsilon_{i}\left(\mathbf{x}_{i}\right)\right| \leq \varepsilon_{i}^{*}, \forall \mathbf{x} \in \Omega_{x}$, where $\varepsilon_{i}^{*} \in \mathbb{R}^{+}$are constants that can be made sufficiently small by increasing $N_{i}$. Applying (10) to (1) yields

$$
\left\{\begin{array}{l}
\dot{x}_{i}=\Phi_{\zeta i}^{T}\left(\mathbf{x}_{i}\right) W_{\zeta i}^{*}+\varepsilon_{i}\left(\mathbf{x}_{i}\right)+x_{i+1} \\
(i=1,2, \cdots, n-1) \\
\dot{x}_{n}=\Phi_{\zeta n}^{T}\left(\mathbf{x}_{n}\right) W_{\zeta n}^{*}+\varepsilon_{n}\left(\mathbf{x}_{n}\right)+u
\end{array} .\right.
$$

Applying (7) to (11) and after some transformations, one obtains the closedloop tracking error dynamics

$$
\left\{\begin{array}{l}
\dot{e}_{i}=v_{i}+e_{i+1}+\Phi_{\zeta i}^{T}\left(\mathbf{x}_{i}\right) \tilde{W}_{\zeta i}+\tilde{\alpha}_{i}+\varepsilon_{i} \\
(i=1,2, \cdots, n-1) \\
\dot{e}_{n}=v_{n}+\Phi_{\zeta n}^{T}\left(\mathbf{x}_{n}\right) \tilde{W}_{\zeta n}+\varepsilon_{n}
\end{array}\right.
$$

where $\tilde{W}_{\zeta i}:=W_{\zeta i}^{*}-\hat{W}_{\zeta i}$ is a parameter estimation error. The detailed steps to obtain (12) can be referred to [30]. 


\subsection{Composite learning using neural networks}

In the composite ANC design, the prediction errors $\epsilon_{i}$ are usually generated by first-order filters as follows [28]:

$$
\dot{\epsilon}_{i}=-\alpha_{f} \epsilon_{i}+\Phi_{i}^{T}\left(\mathbf{x}_{i}\right) \tilde{W}_{i}+\varepsilon_{i}
$$

with $\alpha_{f} \in \mathbb{R}^{+}$being a filtering constant such that $\epsilon_{i}$ can be obtained without the usage of $\dot{x}_{i}$. Although the convergence of both $e_{i}$ and $\epsilon_{i}$ can be achieved in composite ANC, the PE condition still has to be satisfied to guarantee partial convergence of $\tilde{W}_{i}$. In this section, composite learning laws of $\hat{W}_{i}$ are designed such that partial convergence of $\tilde{W}_{i}$ can be guaranteed by the IE condition in Definition 1 which is much weaker than the PE condition in Definition 2.

Let $\frac{\lambda}{s+\lambda}$ be a stable low-pass filter with $s$ a complex variable and $\lambda \in \mathbb{R}^{+}$a filtering constant. To avoid the usage of $\dot{x}_{i}$ in parameter update, $\frac{\lambda}{s+\lambda}$ is applied to each item of (11) such that

$$
\left\{\begin{array}{l}
\chi_{i}=\Phi_{f \zeta i}^{T}(t) W_{\zeta i}^{*}+\varepsilon_{f i}(t)+x_{f(i+1)} \\
(i=1,2, \cdots, n-1) \\
\chi_{n}=\Phi_{f \zeta n}^{T}(t) W_{\zeta n}^{*}+\varepsilon_{f n}(t)+u_{f}
\end{array}\right.
$$

in which $\chi_{i}=\frac{\lambda s}{s+\lambda}\left[x_{i}\right], \Phi_{f \zeta i}=\frac{\lambda}{s+\lambda}\left[\Phi_{\zeta i}\right], \varepsilon_{f i}=\frac{\lambda}{s+\lambda}\left[\varepsilon_{i}\right], x_{f(i+1)}=\frac{\lambda}{s+\lambda}\left[x_{i+1}\right]$ and $u_{f}=\frac{\lambda}{s+\lambda}[u]$ are denoted in hybrid time-frequency domain [45]. Define an excitation matrix

$$
\Theta_{i}(t):=\int_{t-\tau_{d}}^{t} \Phi_{f \zeta i}(\tau) \Phi_{f \zeta i}^{T}(\tau) d \tau .
$$

Multiplying each side of the $i$ th equation in (13) by $\Phi_{f i}$ and integrating the result over $\left[t-\tau_{d}, t\right]$, one obtains

$$
\left\{\begin{array}{l}
\Theta_{i}(t) W_{\zeta i}^{*}+\varepsilon_{a i}(t)=\int_{t-\tau_{d}}^{t} \Phi_{f \zeta i}\left(\chi_{i}-x_{f(i+1)}\right) d \tau \\
(i=1,2, \cdots, n-1) \\
\Theta_{n}(t) W_{\zeta n}^{*}+\varepsilon_{a n}(t)=\int_{t-\tau_{d}}^{t} \Phi_{f \zeta n}\left(\chi_{n}-u_{f}\right) d \tau
\end{array}\right.
$$

with $\varepsilon_{a i}(t):=\int_{t-\tau_{d}}^{t} \Phi_{f \zeta i}(\tau) \varepsilon_{f i}(\tau) d \tau$. Combining the definitions of $\varepsilon_{a i}$ with $\left\|\Phi_{\zeta i}\right\|=\left\|\Phi_{i}\right\| \leq \psi_{i}, \Phi_{f \zeta i}=\frac{\lambda}{s+\lambda}\left[\Phi_{\zeta i}\right],\left|\varepsilon_{i}\right| \leq \varepsilon_{i}^{*}$ and $\varepsilon_{f i}=\frac{\lambda}{s+\lambda}\left[\varepsilon_{i}\right]$, one obtains $\left\|\varepsilon_{a i}\right\| \leq \tau_{d} \psi_{i} \varepsilon_{i}^{*}, \forall \mathbf{x} \in \Omega_{x}$.

From Lemma 2 and Definitions 1 and 2 , for any given $\mathcal{C}^{1}$ trajectory $\mathbf{x}(t)$ that is not necessary to be recurrent, there exist constants $T_{e i}>T_{a}$ and $\sigma_{i} \in \mathbb{R}^{+}$such that $\Phi_{\zeta i}$ is of IE over $\left[T_{e i}-\tau_{d}, T_{e i}\right]$ implying $\Theta_{i}\left(T_{e i}\right) \geq \sigma_{i} I$. Define computable 
prediction errors

$$
\boldsymbol{\epsilon}_{i}(t)=\left\{\begin{array}{l}
\Theta_{i}(t) \tilde{W}_{\zeta i}(t)+\varepsilon_{a i}(t), t<T_{e} \\
\Theta_{i}\left(T_{e i}\right) \tilde{W}_{\zeta i}(t)+\varepsilon_{a i}\left(T_{e i}\right), t \geq T_{e}
\end{array}\right.
$$

with $T_{e}:=\max \left\{T_{e i}\right\}$. Noting $\tilde{W}_{\zeta i}=W_{\zeta i}^{*}-\hat{W}_{\zeta i}$, one obtains $\boldsymbol{\epsilon}_{i}=\left(\Theta_{i} W_{\zeta i}^{*}+\right.$ $\left.\boldsymbol{\varepsilon}_{a i}\right)-\Theta_{i} \hat{W}_{\zeta i}$ where $\left(\Theta_{i} W_{\zeta i}^{*}+\boldsymbol{\varepsilon}_{a i}\right)$ is available via (15) such that $\boldsymbol{\epsilon}_{i}$ in (16) is attainable. Define compact sets $\Omega_{w_{i}}:=\left\{\hat{W}_{\zeta i} \mid\left\|\hat{W}_{\zeta i}\right\| \leq c_{w_{i}}\right\}$, where $c_{w_{i}} \in \mathbb{R}^{+}$ are some constants. Then, design composite update laws

$$
\dot{\hat{W}}_{\zeta i}=\gamma_{i} \mathcal{P}\left(\Phi_{\zeta i}\left(\mathbf{x}_{i}\right) e_{i}+\kappa_{i} \boldsymbol{\epsilon}_{i}\right)
$$

in which $\gamma_{i} \in \mathbb{R}^{+}$are learning rates, $\kappa_{i} \in \mathbb{R}^{+}$are weight factors, and $\mathcal{P}(\bullet)$ is a projection operator given by [42]

$$
\mathcal{P}(\bullet)=\left\{\begin{array}{l}
\bullet, \text { if }\left\|\hat{W}_{\zeta i}\right\|<c_{w_{i}} \text { or }\left\|\hat{W}_{\zeta i}\right\|=c_{w_{i}} \& \hat{W}_{\zeta i}^{T} \bullet \leq 0 \\
\bullet-\frac{\hat{W}_{\zeta i} \hat{W}_{\zeta i}^{T}}{\hat{W}_{\zeta i}^{T} \hat{W}_{\zeta i}} \bullet \text { otherwise }
\end{array} .\right.
$$

Remark 2: Another advantage of applying RCRBF-NNs is that the subregressor $\Phi_{\zeta i}\left(\mathbf{x}_{i}\right)$ activated along any given trajectory $\mathbf{x}(t)$ can be exactly determined due to the compact support of RCRBFs such that the IE condition in Definition 1 is verifiable by checking the minimal singular value of $\Theta_{i}(t)$ in (14) and the time $T_{e i}$ that satisfies the IE condition is obtainable accordingly.

\subsection{Stability and convergence analysis}

The following lemmas are useful in the subsequent analysis.

Lemma $3[39]^{2}$ : Consider the system (1) and Assumptions 1 and 2. For any given $\mathbf{x}(0) \in \Omega_{x_{0}} \subset \mathbb{R}^{n}$, there exist $\Omega_{x} \supset \Omega_{x_{0}}$ and $T_{a} \in \mathbb{R}^{+}$such that $\mathbf{x}(t) \in \Omega_{x}$, $\forall t \in\left[0, T_{a}\right)$.

Lemma 4 [40]: For the filter (9) on $t \in\left[0, T_{a}\right.$ ) under Assumptions 1 and 2, given any small constant $\mu \in \mathbb{R}^{+}$, there exists a sufficiently large frequency $\omega$ in (9) such that $\left|\tilde{\alpha}_{i}(t)\right| \leq \mu, \forall t \in\left[0, T_{a}\right)$.

Let $\hat{W}_{\zeta}:=\left[\hat{W}_{\zeta 1}^{T}, \hat{W}_{\zeta 2}^{T}, \cdots, \hat{W}_{\zeta n}^{T}\right]^{T}, W_{\zeta}^{*}:=\left[W_{\zeta 1}^{* T}, W_{\zeta 2}^{* T}, \cdots, W_{\zeta n}^{* T}\right]^{T}$ and $\tilde{W}_{\zeta}:=$ $W_{\zeta}^{*}-\hat{W}_{\zeta}$. Define compact sets $\Omega_{x}:=\left\{\mathbf{x} \mid\|\mathbf{x}\| \leq c_{x}\right\}, \Omega_{x_{0}}:=\left\{\mathbf{x} \mid\|\mathbf{x}\| \leq c_{x_{0}}\right\}$,

$\overline{2}$ Lemma 3 implies that the state vector $\mathbf{x}$ of the closed-loop system does not have finite escape time during a short interval $\forall t \in\left[0, T_{a}\right)$. 
$\Omega_{e}:=\left\{\mathbf{e} \mid \mathbf{x} \in \Omega_{x}, \mathbf{x}_{d} \in \Omega_{d}\right\}=\left\{\mathbf{e} \mid\|\mathbf{e}\| \leq c_{e}\right\}, \Omega_{e_{0}}:=\left\{\mathbf{e} \mid \mathbf{x} \in \Omega_{x_{0}}, \mathbf{x}_{d} \in \Omega_{d}\right\}=$ $\left\{\mathbf{e} \mid\|\mathbf{e}\| \leq c_{e 0}\right\}$ and $\Omega_{w}:=\prod_{i=1}^{n} \Omega_{w_{i}}$, in which $c_{x}, c_{x_{0}}, c_{e}, c_{e 0} \in \mathbb{R}^{+}$are some constants. As $\Omega_{x} \supset \Omega_{x_{0}}$, one has $c_{x}>c_{x_{0}}$ and $c_{e}>c_{e 0}$. The following theorem demonstrates the main results of this study.

Theorem 1: For the system (1) under Assumption 1 with $\mathbf{x}(0) \in \Omega_{x_{0}}$ and $\boldsymbol{x}_{d}(t)$ under Assumption 2 driven by the control law constituted by (7)-(9) and (17) with $\hat{W}_{\zeta i}(0) \in \Omega_{w i}$, if there exist constants $T_{e i}>T_{a}$ and $\sigma_{i} \in \mathbb{R}^{+}$to satisfy the IE conditions $\Theta_{i}\left(T_{e i}\right) \geq \sigma_{i} I$ in Definition 1 and the control parameters $k_{c i}$ and $\beta_{i}$ in (8) are chosen to satisfy

$$
\begin{array}{r}
k_{c 1}, k_{c n}>1 / 2, k_{c i}>1, i=2 \text { to } n-1, \\
\beta_{n} \geq \varepsilon_{n}^{*}, \beta_{i} \geq \varepsilon_{i}^{*}+\mu, i=1 \text { to } n-1
\end{array}
$$

there exist sufficiently large control parameters $k_{c i}, \gamma_{i}$ and $\omega$ so that all signals involved are of $L_{\infty}$ on $t \in[0, \infty)$ and the equilibrium point of the closed-loop system has practical exponential stability on $t \in\left[T_{e}, \infty\right)$.

Proof: Firstly, noting Lemma 3, one gets $\mathbf{x}(t) \in \Omega_{x}$ so that $\mathbf{e}(t) \in \Omega_{e}$ on $t \in\left[0, T_{a}\right), \forall \mathbf{x}(0) \in \Omega_{x_{0}}$. Noting Lemma 4 , one has $\left|\tilde{\alpha}_{i}(t)\right| \leq \mu, \forall t \in\left[0, T_{a}\right)$. Choose a Lyapunov function candidate

$$
V_{i}\left(e_{i}, \tilde{W}_{\zeta i}\right)=e_{i}^{2} / 2+\tilde{W}_{\zeta i}^{T} \tilde{W}_{\zeta i} /\left(2 \gamma_{i}\right)
$$

for the $i$ th subsystem of (12). Differentiating $V_{i}$ in (20) along (12) with respect to time $t$ and using (8), one gets

$$
\begin{aligned}
\dot{V}_{i}= & -k_{c i} e_{i}^{2}+e_{i} e_{i+1}-\beta_{i} e_{i} \tanh \left(e_{i} / v_{i}\right) \\
& +e_{i}\left(\varepsilon_{i}+\tilde{\alpha}_{i}\right)+\tilde{W}_{\zeta i}^{T}\left(\Phi_{\zeta i}\left(\mathbf{x}_{i}\right) e_{i}-\dot{\hat{W}}_{\zeta i} / \gamma_{i}\right)
\end{aligned}
$$

with $\tilde{\alpha}_{n}=e_{n+1}=0$. From the projection operation result in [42, Th. 4.6.1], $\mathcal{P}$ in (17) guarantees $\hat{W}_{\zeta i}(t) \in \Omega_{w_{i}}, \forall t \geq 0$ and

$$
\tilde{W}_{\zeta i}^{T}\left(\Phi_{\zeta i}\left(\mathbf{x}_{i}\right) e_{i}-\dot{\hat{W}}_{\zeta i} / \gamma_{i}\right) \leq-\kappa_{i} \tilde{W}_{\zeta i}^{T} \boldsymbol{\epsilon}_{i}
$$

as long as $\hat{W}_{\zeta i}(0) \in \Omega_{w_{i}}$. Applying the above result to (21) and noting $\left|\varepsilon_{i}\right| \leq$ $\varepsilon_{i}^{*}$ and $\left|\tilde{\alpha}_{i}\right| \leq \mu$, one obtains

$$
\begin{aligned}
\dot{V}_{i} \leq & -k_{c i} e_{i}^{2}+e_{i} e_{i+1}-\kappa_{i} \tilde{W}_{\zeta i}^{T} \boldsymbol{\epsilon}_{i} \\
& +\left(\varepsilon_{i}^{*}+\mu\right)\left|e_{i}\right|-\beta_{i} e_{i} \tanh \left(e_{i} / v_{i}\right) .
\end{aligned}
$$

Applying $0 \leq\left|e_{i}\right|-e_{i} \tanh \left(e_{i} / v_{i}\right) \leq c_{\kappa} v_{i}$ with $c_{\kappa}=0.2785$ [1] to the above result and noting the selection of $\beta_{i}$ in (19) yields

$$
\dot{V}_{i} \leq-k_{c i} e_{i}^{2}+e_{i} e_{i+1}+c_{\kappa} \beta_{i} v_{i}-\kappa_{i} \tilde{W}_{\zeta i}^{T} \boldsymbol{\epsilon}_{i}
$$


Applying the formulas of $\boldsymbol{\epsilon}_{i}$ in (16) to the above result yields

$$
\begin{aligned}
\dot{V}_{i} \leq & -k_{c i} e_{i}^{2}+e_{i} e_{i+1}+c_{\kappa} \beta_{i} v_{i} \\
& -\kappa_{i} \tilde{W}_{\zeta i}^{T} \Theta_{i} \tilde{W}_{\zeta i}-\kappa_{i} \tilde{W}_{\zeta i}^{T} \varepsilon_{a i} .
\end{aligned}
$$

Secondly, the stability is analyzed at $t \in[0, \infty)$. Applying $\Theta_{i} \geq 0$ from (14) and $e_{i} e_{i+1} \leq e_{i}^{2} / 2+e_{i+1}^{2} / 2$ to $(22)$, one gets

$$
\dot{V}_{i} \leq-k_{s i} e_{i}^{2} / 2+c_{\kappa} \beta_{i} v_{i}-\kappa_{i} \tilde{W}_{\zeta i}^{T} \varepsilon_{a i}
$$

with $k_{s 1}:=2 k_{c 1}-1, k_{s n}:=2 k_{c n}-1$ and $k_{s i}:=2 k_{c i}-2$ for $i=2$ to $n-1$. from the above result, one obtains

$$
\begin{aligned}
\dot{V}_{i} \leq & -k_{s i}\left(e_{i}^{2} / 2+\tilde{W}_{\zeta i}^{T} \tilde{W}_{\zeta i} /\left(2 \gamma_{i}\right)\right)+c_{\kappa} \beta_{i} v_{i} \\
& +k_{s i} \tilde{W}_{\zeta i}^{T} \tilde{W}_{\zeta i} /\left(2 \gamma_{i}\right)-\kappa_{i} \tilde{W}_{\zeta i}^{T} \varepsilon_{a i} .
\end{aligned}
$$

Noting (20), the above result leads to

$$
\dot{V}_{i} \leq-k_{s i} V_{i}(t)+k_{s i} \tilde{W}_{\zeta i}^{T} \tilde{W}_{\zeta i} /\left(2 \gamma_{i}\right)-\kappa_{i} \tilde{W}_{\zeta i}^{T} \varepsilon_{a i}+c_{\kappa} \beta_{i} v_{i}
$$

Noting $\hat{W}_{\zeta i}, W_{\zeta i}^{*} \in \Omega_{w_{i}}, \Omega_{w_{i}}=\left\{\hat{W}_{\zeta i} \mid\left\|\hat{W}_{\zeta i}\right\| \leq c_{w_{i}}\right\}$ and $\tilde{W}_{\zeta i}=W_{\zeta i}^{*}-\hat{W}_{\zeta i}$, one gets $\left\|\tilde{W}_{\zeta i}\right\| \leq 2 c_{w i}$. Substituting $\left\|\tilde{W}_{\zeta i}\right\| \leq 2 c_{w i}$ and $\left\|\varepsilon_{a i}\right\| \leq \tau_{d} \psi_{i} \varepsilon_{i}^{*}, \forall \mathbf{x} \in \Omega_{x}$ into the foregoing result, one gets

$$
\dot{V}_{i} \leq-k_{s i} V_{i}+2 k_{s i} c_{w_{i}}^{2} / \gamma_{i}+2 \tau_{d} \kappa_{i} c_{w_{i}} \psi_{i} \varepsilon_{i}^{*}+c_{\kappa} \beta_{i} v_{i}
$$

Applying [42, Lemma A.3.2] to the above inequality yields

$$
V_{i}(t) \leq\left(V_{i}(0)-\eta_{i}\right) e^{-k_{s i} t}+\eta_{i}, \forall t \in\left[0, T_{a}\right)
$$

on $\mathbf{x} \in \Omega_{x}$ where $\eta_{i}\left(k_{c i}, \gamma_{i}, \varepsilon_{\zeta i}^{*}\right):=2 c_{w_{i}}^{2} / \gamma_{i}+2 \tau_{d} \kappa_{i} c_{w_{i}} \psi_{i} \varepsilon_{i}^{*} / k_{s i}+c_{\kappa} \beta_{i} v_{i} / k_{s i}$, implying $V_{i}(t) \leq \max \left\{\eta_{i}, V_{i}(0)\right\}$. Let $V:=\sum_{i=1}^{n} V_{i}$ be a Lyapunov function candidate of the entire system so that

$$
V(t) \leq V(0) e^{-k_{s} t}+\eta, \forall t \in\left[0, T_{a}\right)
$$

on $\mathbf{x} \in \Omega_{x}$ with $\eta:=\sum_{i=1}^{n} \eta_{i} \in \mathbb{R}^{+}$and $k_{s}:=\min _{i \in[1, n]}\left\{k_{s i}\right\} \in \mathbb{R}^{+}$, where the positivity of $k_{s}$ is from the definitions of $k_{s i}$ and the choice of $k_{c i}$ in (18). The conditions $\mathbf{x} \in \Omega_{x}$ (implying $\mathbf{e} \in \Omega_{e}$ ) and $\hat{W}_{i}, W_{i}^{*} \in \Omega_{w_{i}}$ are used to determine a Lyapunov surface

$$
V=\delta, \delta:=c_{e}^{2} / 2+\sum_{i=1}^{n} 2 c_{w_{i}}^{2} / \gamma_{i}
$$


Thus, one has $\delta>V(0)$ as $c_{e 0}<c_{e}$ and $\hat{W}_{\zeta i}(0) \in \Omega_{w_{i}}$. Then, there exist sufficiently large $k_{c i}$ and $\omega$ to satisfy

$$
\sum_{i=1}^{n}\left(2 \tau_{d} \kappa_{i} c_{w_{i}} \psi_{i} \varepsilon_{i}^{*} / k_{s i}+c_{\kappa} \beta_{i} v_{i} / k_{s i}\right)<c_{e}^{2} / 2
$$

implying $\eta<\delta$. Thus, $\{V \leq \delta\} \cap \Omega_{w}$ is positively invariant such that the trajectories of $\left(\mathbf{e}(t), \tilde{W}_{\zeta}(t)\right)$ started from $\Omega_{e 0} \cap \Omega_{w}$ stay within $\{V \leq \delta\} \cap$ $\Omega_{w}$ for all time implying $T_{a}=\infty$ and converge to $\{V \leq \eta\} \cap \Omega_{w}$ as $t \rightarrow \infty$. Hence, one gets $\mathbf{e}(t) \in \Omega_{e}, \tilde{W} \zeta(t) \in \Omega_{w}, \forall t \in[0, \infty)$ implying $\mathbf{x}(t), u(t), \alpha_{i}(t)$, $\alpha_{i}^{c}(t) \in L_{\infty}, \forall t \in[0, \infty)$. Consequently, all signals involved are of $L_{\infty}$ on $t \in$ $[0, \infty)$.

Thirdly, the stability is analyzed at $t \in\left[T_{e}, \infty\right)$. As there exist $T_{e i}>T_{a}$ and $\sigma_{i} \in \mathbb{R}^{+}$to satisfy $\Theta_{i}\left(T_{e i}\right) \geq \sigma_{i} I$, it follows from (22) with the derivations in the second part that

$$
\begin{aligned}
\dot{V}_{i} \leq & -k_{s i} e_{i}^{2} / 2-\kappa_{i} \sigma_{i} \tilde{W}_{\zeta i}^{T} \tilde{W}_{\zeta i}-\kappa_{i} \tilde{W}_{\zeta i}^{T} \varepsilon_{a i}+c_{\kappa} \beta_{i} v_{i} \\
= & -k_{s i} e_{i}^{2} / 2-\kappa_{i} \sigma_{i} \tilde{W}_{\zeta i}^{T} \tilde{W}_{\zeta i} / 2+c_{\kappa} \beta_{i} v_{i} \\
& -\kappa_{i} \sigma_{i}\left(\tilde{W}_{\zeta i}^{T} \tilde{W}_{\zeta i}+2 \tilde{W}_{\zeta i}^{T} \varepsilon_{a i} / \sigma_{i}\right) / 2
\end{aligned}
$$

Applying the Young's inequality to the third line of the above expression and noting (20) and $\left\|\varepsilon_{a i}\right\| \leq \tau_{d} \psi_{i} \varepsilon_{i}^{*}$, one gets

$$
\dot{V}_{i} \leq-k_{m i} V_{i}(t)+c_{\kappa} \beta_{i} v_{i}+\kappa_{i}\left(\tau_{d} \psi_{i} \varepsilon_{i}^{*}\right)^{2} /\left(2 \sigma_{i}\right)
$$

with $k_{m i}:=\min \left\{k_{s i}, \gamma_{i} \kappa_{i} \sigma_{i}\right\}$. Thus, one gets

$$
\dot{V}_{i}(t) \leq-k_{m i} V_{i}(t)+k_{m i} \varphi_{i}, \forall t \in\left[T_{e}, \infty\right)
$$

in which $\varphi_{i}\left(k_{c i}, \gamma_{i}, \omega, \varepsilon_{i}^{*}\right):=\kappa_{i}\left(\tau_{d} \psi_{i} \varepsilon_{i}^{*}\right)^{2} /\left(2 k_{m i} \sigma_{i}\right)+c_{\kappa} \beta_{i} v_{i} / k_{m i}$. Solving the above inequality using [42, Lemma A.3.2] leads to

$$
V_{i}(t) \leq\left(V_{i}\left(T_{e}\right)-\varphi_{i}\right) e^{-k_{m i} t}+\varphi_{i}, \forall t \in\left[T_{e}, \infty\right) .
$$

Using the above result, one obtains

$$
V(t) \leq V\left(T_{e}\right) e^{-k_{m} t}+\varphi, \forall t \in\left[T_{e}, \infty\right)
$$

with $\varphi:=\sum_{i=1}^{n} \varphi_{i} \in \mathbb{R}^{+}$and $k_{m}:=\min _{1 \leq i \leq n}\left\{k_{m i}\right\} \in \mathbb{R}^{+}$, which implies the trajectories of $\left(\mathbf{e}(t), \tilde{W}_{\zeta}(t)\right)$ exponentially converge to a positively invariant set $\{V \leq \varphi\}$. Thus, practical exponential stability is achieved on $t \in\left[T_{e}, \infty\right)$ in the sense that $\mathbf{e}(t)$ and $\tilde{W}_{\zeta}(t)$ converge to small neighborhoods of $\mathbf{0}$ dominated by $k_{c i}, \gamma_{i}$ and $\omega$.

\section{END}


Remark 3: Dynamic regressor extension and mixing (DREM) is an alternative parameter estimation approach where the PE condition of the regressor $\Phi_{f}$ is relaxed to be a non-square-integrability condition for the determinant of an instrumental matrix [46]. However, the DREM estimator was studied only for open-loop parameter estimation and its closed-loop stability was not formally proven. In addition, the non-square-integrability condition is not directly correlated to the IE condition so that parameter convergence still may not be guaranteed for the DREM estimator even if the IE condition is satisfied.

\section{$5 \quad$ Illustrative Results}

Consider the following model that describes aircraft wing rock [28]:

$$
\left\{\begin{array}{l}
\dot{x}_{1}=x_{2} \\
\dot{x}_{i}=f_{2}\left(\mathbf{x}_{2}\right)+b_{2} x_{3} \\
\dot{x}_{n}=-b_{3} x_{3}+b_{3} u
\end{array}\right.
$$

where $x_{1}(\mathrm{rad})$ is the aircraft roll angle, $x_{2}(\mathrm{rad} / \mathrm{s})$ is the roll rate, $x_{3}(\mathrm{~N})$ is the actuator output, $b_{2}, b_{3} \in \mathbb{R}^{+}$are known constants, and $f_{2}\left(\mathbf{x}_{2}\right)$ is an unknown function. For simulation, set $b_{2}=3, b_{3}=15, f_{2}\left(\mathbf{x}_{2}\right)=0.2314 x_{1}+0.6918 x_{2}-$ $0.6245\left|x_{1}\right| x_{2}+0.0095\left|x_{2}\right| x_{2}+0.0214 x_{1}^{3}+0.8, c_{w}=5$, and $\mathbf{x}(0)=[\pi / 6,0,0]^{T}$. From (23), only one RCRBF-NN $\hat{f}_{2}\left(\mathbf{x}_{2}, \hat{W}_{2}\right)$ in (6) is needed to approximate $f_{2}\left(\mathbf{x}_{2}\right)$.

The control law composed of (7)-(9) and (17) is set up as follows: 1) let the domain $\Omega_{x}=[-1.2,1.2] \times[-0.6,0.6]$ and select RCRBFs with $m_{1}=m_{2}=7$, $\sigma_{1}=0.6$ and $\sigma_{2}=0.3$ to evenly cover $\Omega_{x}$ so that one has $N=7^{2}=49 \mathrm{NN}$ nodes in total; 2) set the control parameters $k_{1}=k_{2}=2, k_{3}=1, v_{1}=v_{2}=$ $v_{3}=0.1, \beta_{1}=0.2, \beta_{2}=0.1, \beta_{3}=0$ in $\left.(8) ; 3\right)$ set the filter parameters $\omega=$ 20 and $\varsigma=1$ in (9) and $\lambda=30$ in (13); 4) set the integral duration $\tau_{d}=25$ in (14); 5) set the learning parameters $\gamma_{2}=10$ and $\kappa_{2}=30$ in (17). Simulation is carried out in MATLAB software with the solver being fixed-step ode 1 and the step size being $1 \mathrm{~ms}$. In addition, $35 \mathrm{~dB}$ Gaussian white noise is applied to corrupt state measurement, and the traditional NNLC with $\kappa_{2}=0$ is selected as the baseline controller.

The reference output $\mathbf{x}_{d}$ is generated by

$$
\dot{\mathbf{x}}_{d}=\left[\begin{array}{cc}
0 & 1 \\
-1 & -21
\end{array}\right] \mathbf{x}_{d}+\left[\begin{array}{l}
0 \\
1
\end{array}\right] x_{c}
$$



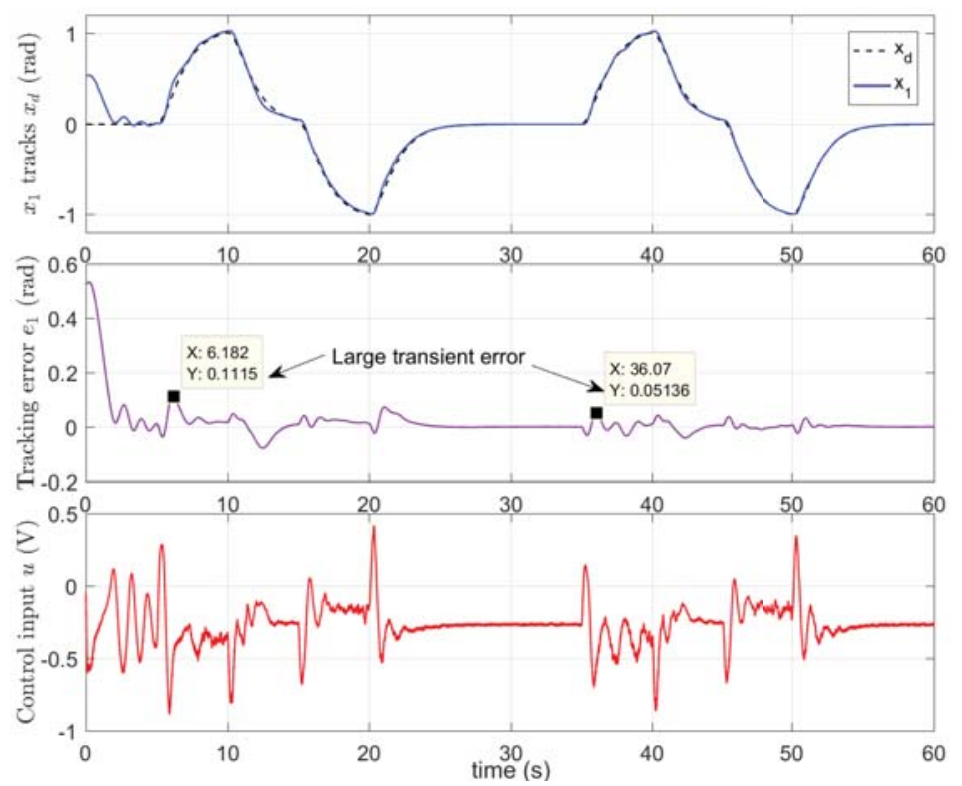

(a)
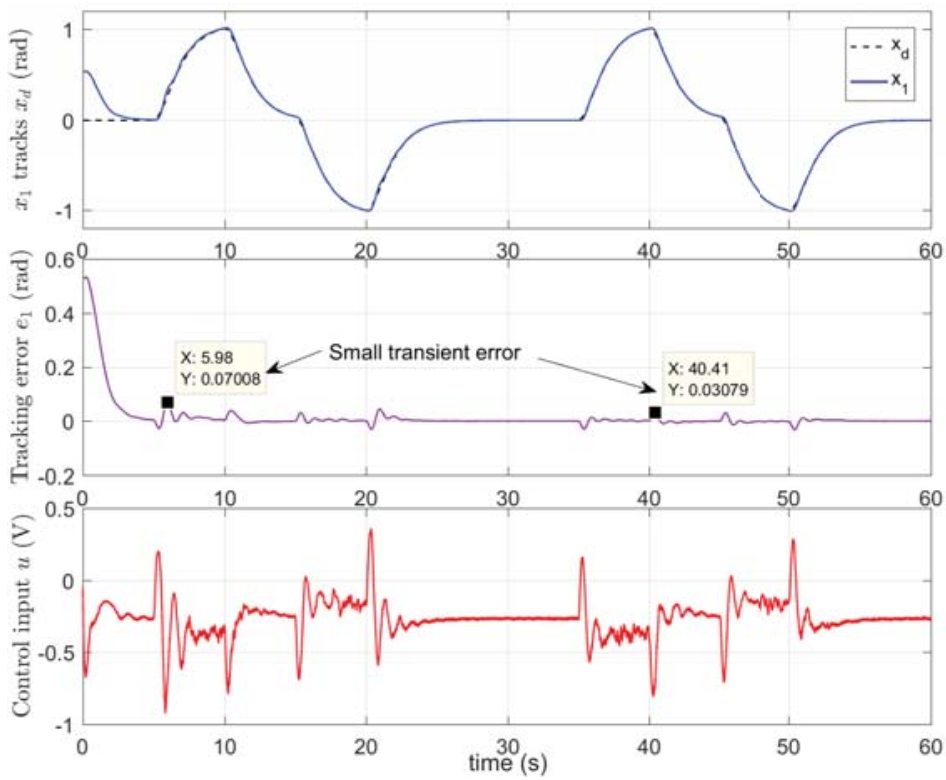

(b)

Fig. 2. Control performances under two controllers. (a) By the NNLC. (b) By the proposed NNCLC.

where $x_{c}=\pi / 3$ at $t \in[5,10] \cup[35,40] \mathrm{s}, x_{c}=-\pi / 3$ at $t \in[15,20] \cup[45,50]$ $\mathrm{s}$ and $x_{c}=0$ for other time. It is clear that the $\mathbf{x}_{d}$ generated by the above model includes two tasks that are the same, and it does not satisfy the partial $\mathrm{PE}$ condition in Lemma 2. Control trajectories by the two controllers are depicted in Fig. 2. It is shown that the proposed NNCLC achieves a much 


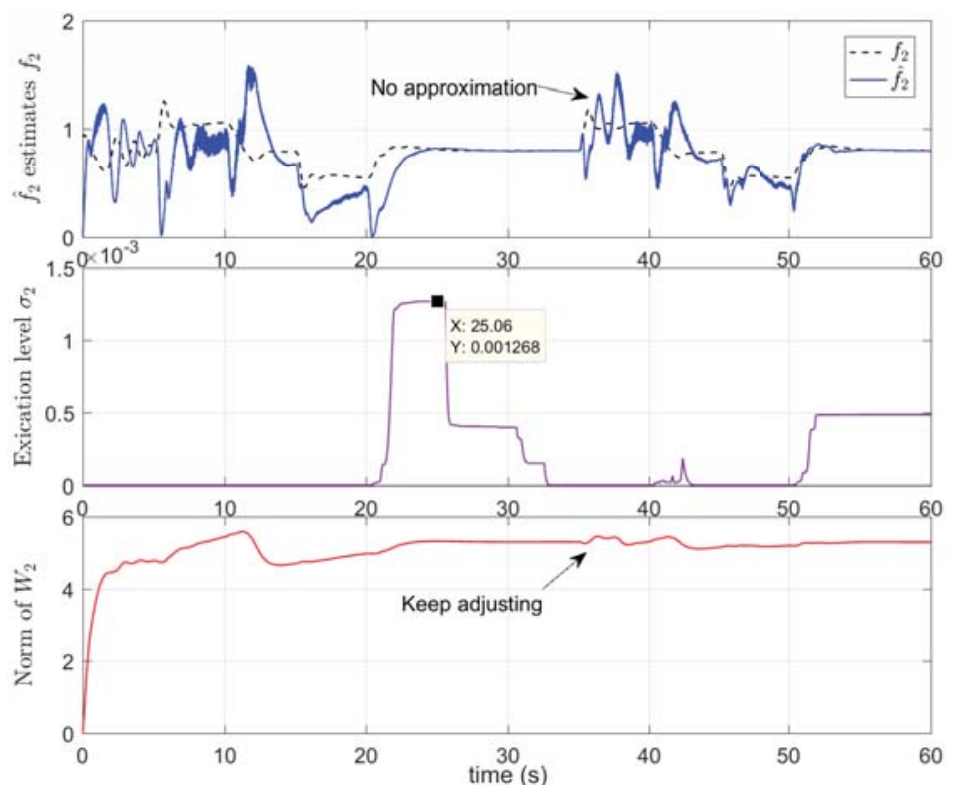

(a)

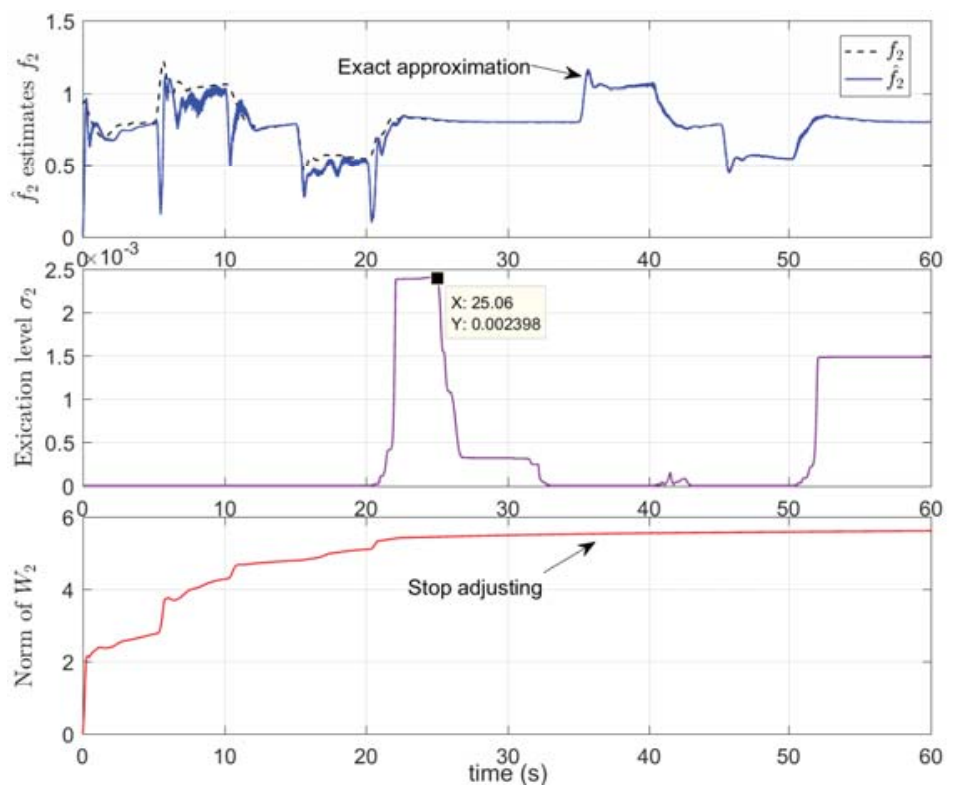

(b)

Fig. 3. Learning performances under two controllers. (a) By the NNLC. (b) By the proposed NNCLC.

better transient tracking performance than the NNLC under the control input $u$ with a similar gain and less oscillations, where the transient errors $e_{1}$ by the proposed NNCLC are reduced from 0.1115 and 0.05136 to 0.07008 and 0.03079 for the first and second tasks, respectively. The slight oscillations in $u$ result from the significantly and frequently changing reference trajectory $\mathbf{x}_{d}$. 
Learning trajectories under the two controllers are depicted in Fig. 3. For the NNLC, no approximation of $f_{2}$ and no convergence of $\left\|\hat{W}_{2}\right\|$ are shown due to the absence of the partial PE condition. On the contrary, for the proposed NNCLC, $f_{2}$ is accurately estimated and $\left\|\hat{W}_{2}\right\|$ converges to a constant after a short transient process. The excitation level $\sigma_{2}$ based on the subregressor $\Phi_{f \zeta 2}$ is clearly shown in Fig. 3, where $\sigma_{2}$ by the proposed NNCLC is about two times larger than that by the NNLC. The major drawbacks of the proposed NNCLC include: 1) The calculation of the prediction errors $\boldsymbol{\epsilon}_{i}$ in (16) with $i=$ 1 to $n$ increases the computational cost; 2 ) the composite update laws (17) with $i=1$ to $n$ are more sensitive to external disturbances so that the control design needs to be carefully considered in this case.

\section{Conclusions}

In this brief, an NNCLC strategy based on RCRBF-NNs has been developed for a class of strict-feedback nonlinear systems with mismatched uncertainties, where closed-loop practical exponential stability is established under the IE condition that relaxes the classical PE condition. Compared with existing composite learning approaches, the proposed approach has two distinctive features: 1) An exact estimation of plant uncertainties is achieved without using the time derivatives of plant states; 2) a subregressor activated along any trajectory of NN inputs can be determined such that the IE condition is verifiable. Illustrative results have demonstrated that the proposed NNCLC achieves much better control and learning performances under a similar control input compared with the traditional NNLC, and the excitation level is clearly shown due to the usage of RCRBF-NNs. The determination of centers and widths of RBFs using self-organizing techniques [47-49] and the extension to a more general class of pure-feedback nonlinear systems [50] for the proposed approach are interesting for future studies.

\section{References}

[1] S. S. Ge, C. C. Hang, T. H. Lee, and T. Zhang, Stable Adaptive Neural Network Control. New York, NY, USA: Springer, 2002.

[2] K. S. Narendra, "Neural networks for control: Theory and practice," Proc. IEEE, vol. 84, no. 10, pp. 1385-1406, Oct. 1996.

[3] F. F. M. El-Sousy, "Adaptive hybrid control system using a recurrent RBFNbased self-evolving fuzzy-neural-network for PMSM servo drives," Applied Soft Comput., vol. 21, pp. 509-532, Aug. 2014. 
[4] A. Loria, G. Espinosa-Perez, and S. Avila-Becerril, "Global adaptive linear control of the permanent-magnet synchronous motor," Int. J. Adaptive Control Signal Process., vol. 28, no. 10, pp. 971-986, Oct. 2014.

[5] Y. P. Pan, H. Y. Yu, and M. J. Er, "Adaptive neural PD control with semiglobal asymptotic stabilization guarantee," IEEE Trans. Neural Netw. Learn. Syst., vol. 25, no. 12, pp. 2264-2274, Dec. 2014.

[6] Z. Y. Chen, "A novel adaptive control approach for nonlinearly parameterized systems," Int. J. Adaptive Control Signal Process., vol. 29, no. 1, pp. 81-98, Jan. 2015.

[7] K. Esfandiari, F. Abdollahi, and H. A. Talebi, "Adaptive control of uncertain nonaffine nonlinear systems with input saturation using neural networks," IEEE Trans. Neural Netw. Learn. Syst., vol. 26, no. 10, pp. 2311-2322, Oct. 2015.

[8] Y. P. Pan, T. R. Sun, and H. Y. Yu, "Peaking-free output-feedback adaptive neural control under a nonseparation principle," IEEE Trans. Neural Netw. Learn. Syst., vol. 26, no. 12, pp. 3097-3108, Dec. 2015.

[9] Y. P. Pan, Y. Q. Liu, B. Xu, and H. Y. Yu, "Hybrid feedback feedforward: An efficient design of adaptive neural network control," Neural Netw., vol. 76, pp. 122-134, Apr. 2016.

[10] F. F. M. El-Sousy and K. A. Abuhasel, "Self-organizing recurrent fuzzy wavelet nural network-based mixed $\mathrm{H}-2 / \mathrm{H}-\infty$ adaptive tracking control for uncertain two-axis motion control system," IEEE Trans. Ind. Appl., vol. 52, no. 6, pp. 5139-5155, Nov. 2016.

[11] R. D. Tehrani, F. Shabaninia, A. Khayatian, and M. H. Asemani, "Transient performance improvement in indirect model reference adaptive control using perturbation-based extremum seeking identifier," Int. J. Adaptive Control Signal Process., vol. 31, no. 8, pp. 1152-1161, Aug. 2017.

[12] M. Hamdy, S. Abd-Elhaleem, and M. Fkirin, "Time-varying delay compensation for a class of nonlinear control systems over network via $H^{\infty}$ adaptive fuzzy controller," IEEE Trans. Syst. Man Cybern.: Syst., vol. 47, no. 8, pp. 21142124, Aug. 2017.

[13] C. Yang, Y. Jiang, Z. Li, W. He, and C. Y. Su, "Neural control of bimanual robots with guaranteed global stability and motion precision," IEEE Trans. Ind. Inf., vol. 13, no. 3, pp. 1162-1171, Jun. 2017.

[14] R. Kumar, S. Srivastava, J. R. P. Gupta, and A. Mohindru, "Self-recurrent wavelet neural network-based identification and adaptive predictive control of nonlinear dynamical systems," Int. J. Adaptive Control Signal Process., vol. 32, no. 9, pp. 1326-1358, Sep. 2018.

[15] J. D. Rubio, "Discrete time control based in neural networks for pendulums," Applied Soft Comput., vol. 68, pp. 821-832, Jul 2018. 
[16] S. Li, H. Q. Wang, and M. U. Rafique, "A novel recurrent neural network for manipulator control with improved noise tolerance," IEEE Trans. Neural Netw. Learn. Syst., vol. 29, no. 5, pp. 1908-1918, May 2018.

[17] L. Liu, Z. S. Wang, X. S. Yao and H. G. Zhang, "Echo state networksbased data-driven adaptive fault tolerant control with its application to electromechanical system," IEEE/ASME Trans. Mechatronics, vol. 23, no. 3, pp. 1372-1382, Jun. 2018.

[18] A. J. Kurdila, F. J. Narcowich, and J. D. Ward, "Persistancy of excitation in identification using radial basis function approximants," SIAM J. Control Optimization, vol. 33, no. 2, pp. 625-642, Mar. 1995.

[19] J. A. Farrell, "Stability and approximator convergence in nonparametric nonlinear adaptive control," IEEE Trans. Neural Netw., vol. 9, no. 5, pp. 10081020, Sep. 1998.

[20] G. Chowdhary, M. Muhlegg, and E. Johnson, "Exponential parameter and tracking error convergence guarantees for adaptive controllers without persistency of excitation," Int. J. Control, vol. 87, no. 8, pp. 1583-1603, May 2014 .

[21] C. Wang and D. J. Hill, "Learning from neural control," IEEE Trans. Neural Netw., vol. 17, no. 1, pp. 130-146, Jan. 2006.

[22] C. Wang, M. Wang, T. F. Liu, and D. J. Hill, "Learning from ISS-modular adaptive NN control of nonlinear strict-feedback systems," IEEE Trans. Neural Netw. Learn. Syst., vol. 23, no. 10, pp. 1539-1550, Oct. 2012.

[23] B. Xu, C. Yang, and Z. Shi, "Reinforcement learning output feedback NN control using deterministic learning technique," IEEE Trans. Neural Netw. Learn. Syst., vol. 25, no. 3, pp. 635-641, Mar 2014.

[24] M. Wang, C. Wang, P. Shi, and X. P. Liu, "Dynamic learning from neural control for strict-feedback systems with guaranteed predefined performance," IEEE Trans. Neural Netw. Learn. Syst., vol. 27, no. 12, pp. 2564-2576, Dec. 2016.

[25] Y. P. Pan and H. Y. Yu, "Biomimetic hybrid feedback feedforward neuralnetwork learning control," IEEE Trans. Neural Netw. Learn. Syst., vol. 28, no. 6, pp. 1481-1487, Jun. 2017.

[26] T. Zheng and C. Wang, "Relationship between persistent excitation levels and RBF network structures, with application to performance analysis of deterministic learning," IEEE Trans. Cybern., to be published.

[27] L. Hsu and R. R. Costa, "Adaptive control with discontinuous $\sigma$-factor and saturation for improved robustness," Int. J. Control, vol. 45, no. 3, pp. 843859, Mar. 1987.

[28] Y. P. Pan, Y. Zhou, T. R. Sun, and M. J. Er, "Composite adaptive fuzzy $H^{\infty}$ tracking control of uncertain nonlinear systems," Neurocomputing, vol. 99, pp. 15-24, Jan. 2013. 
[29] B. Xu, Z. K. Shi, C. G. Yang, and F. C. Sun, "Composite neural dynamic surface control of a class of uncertain nonlinear systems in strict-feedback form," IEEE Trans. Cybern., vol. 44, no. 12, pp. 2626-2634, Dec. 2014.

[30] Y. P. Pan, T. R. Sun, and H. Y. Yu, "Composite adaptive dynamic surface control using online recorded data," Int. J. Robust and Nonlinear Control, vol. 26, no. 18, pp. 3921-3936, Dec. 2016.

[31] Y. P. Pan, J. Zhang, and H. Y. Yu, "Model reference composite learning control without persistency of excitation," IET Control Theory Appl., vol. 10, no. 16, pp. 1963-1971, Oct. 2016.

[32] Y. P. Pan and H. Y. Yu, "Composite learning from adaptive dynamic surface control," IEEE Trans. Autom. Control, vol. 61, no. 9, pp. 2603-2609, Sep. 2016.

[33] Y. P. Pan, T. R. Sun, Y. Q. Liu, and H. Y. Yu, "Composite learning from adaptive backstepping neural network control," Neural Netw., vol. 95, pp. 134142, Nov. 2017.

[34] Y. P. Pan, T. R. Sun, and H. Y. Yu, "On parameter convergence in leastsquares identification and adaptive control," Int. J. Robust Nonlinear Control, to be published, https://doi.org/10.1002/rnc.4527.

[35] Y. P. Pan, S. Aranovskiy, A. Bobtsov, and H. Y. Yu, "Efficient learning from adaptive control under sufficient excitation," Int. J. Robust Nonlinear Control, to be published.

[36] Y. P. Pan and H. Y. Yu, "Composite learning robot control with guaranteed parameter convergence," Automatica, vol. 89, pp. 398-406, Mar. 2018.

[37] K. Guo, Y. P. Pan, and H. Y. Yu, "Composite learning robot control with friction compensation: A neural network-based approach," IEEE Trans. Ind. Electron., to be published, https://doi.org/10.1109/TIE.2018.2886763.

[38] J. A. Farrell, M. Polycarpou, M. Sharma, and W. J. Dong, "Command filtered backstepping," IEEE Trans. Autom. Control, vol. 54, no. 6, pp. 1391-1395, Jun. 2009 .

[39] W. Dong, J. A. Farrell, M. M. Polycarpou, V. Djapic, and M. Sharma, "Command filtered adaptive backstepping," IEEE Trans. Control Syst. Tech., vol. 20, no. 3, pp. 566-580, May. 2012.

[40] J. C. Hu and H. H. Zhang, "Immersion and invariance based command-filtered adaptive backstepping control of VTOL vehicles," Automatica, vol. 49, no. 7, pp. 2160-2167, Jul. 2013.

[41] Y. P. Pan, H. M. Wang, X. Li, and H. Y. Yu, "Adaptive command-filtered backstepping control of robot arms with compliant actuators," IEEE Trans. Control Syst. Tech., vol. 26, no. 3, pp. 1149-1156, May 2018.

[42] J. A. Farrell and M. M. Polycarpou, Adaptive Approximation Based Control: Unifying Neural, Fuzzy and Traditional Adaptive Approximation Approaches. Hoboken, NJ, USA: Wiley, 2006. 
[43] R. J. Schilling, J. J. Carroll, and A. F. Al-Ajlouni, "Approximation of nonlinear systems with radial basis function neural networks," IEEE Trans. Neural Netw., vol. 12, no. 1, pp. 1-15, Jan. 2001.

[44] J. Park and I. W. Sandberg, "Universal approximation using radial-basisfunction networks," Neural Computation, vol. 3, no. 2, pp. 246-257, 1991.

[45] S. S. Sastry and A. Isidori, "Adaptive control of linearizable systems," IEEE Trans. Autom. Control, vol. 34, no. 11, pp. 1123-1131, Nov. 1989.

[46] S. Aranovskiy, A. Bobtsov, R. Ortega, and A. Pyrkin, "Performance enhancement of parameter estimators via dynamic regressor extension and mixing," IEEE Trans. Autom. Control, vol. 62, no. 7, pp. 3546-3550, Jul. 2017.

[47] M. Pratama, S. G. Anavatti, P. P. Angelov, and E. Lughofer, "PANFIS: A novel incremental learning machine," IEEE Trans. Neural Netw. Learn. Syst., vol. 25, no. 1, pp. 55-68, Jan. 2014.

[48] M. Pratama, S. G. Anavatti, and E. Lughofer, "GENEFIS: Toward an effective localist network," IEEE Trans. Fuzzy Syst., vol. 22, no. 3, pp. 547-562, Jun. 2014 .

[49] M. Pratama, P. P. Angelov, E. Lughofer, and M. J. Er, "Parsimonious random vector functional link network for data streams," Information Science, vol. 430, pp. 519-537, Mar. 2018.

[50] M. Krstic, I. Kanellakopoulos, and P. V. Kokotovic, Nonlinear and Adaptive Control Design. New York. NY, USA: Wiley, 1995. 